\title{
MiR-I0I Protects Against the Cerebral I/R Injury Through Regulating JAK2/STAT3 Signaling Pathway
}

\author{
Xiaowang Guo' \\ Xiaoyan Shen ${ }^{2}$ \\ Zhijun Yong ${ }^{3}$
}

'Department Emergency Medicine, Shaanxi Provincial People's Hospital, Xi'an City, Shaanxi Province, 710068, People's Republic of China; ${ }^{2}$ Department of Neurology Medicine, The Fourth People's Hospital of Shaanxi, Xi'an City, Shaanxi Province, 710000, People's Republic of China; ${ }^{3}$ Department of Rehabilitation Medicine, Shaanxi Provincial People's Hospital, Xi'an City, Shaanxi Province, 710068, People's Republic of China
Correspondence: Zhijun Yong Department of Rehabilitation Medicine, Shaanxi Provincial People's Hospital, No. 256 Youyi West Road, Xi'an City, Shaanxi Province, 710068, People's Republic of China

Email zhijunyongmedicine@163.com
Background: Ischemic stroke is a devastating disease with very limited therapeutics. Although miR-101 has been reported to play crucial roles in various human diseases, its role in ischemic stroke remains unclear.

Methods: Ischemia-reperfusion (I/R) injury neuronal cells and rat model with $I / R$ injury were constructed. Viability and apoptosis of I/R model cells with miR-101 overexpression or downregulation were evaluated. Potential targets of miR-101 were predicted using miRNA database microRNA.org and confirmed using luciferase reporter assays. Meanwhile, JAK2 and p-STAT3 protein levels were evaluated by Western blot. In addition, rescue experiments (silencing of JAK2) were applied to determine the role of miR-101 in cerebral $\mathrm{I} / \mathrm{R}$ injury.

Results: MiR-101 was significantly downregulated in OGD/R-induced neuronal cells and brain tissues with $\mathrm{I} / \mathrm{R}$ injury. MiR-101 overexpression (miR-101 mimics) significantly promoted viability and inhibited apoptosis of OGD/R-induced neuronal cells in vitro and efficiently protected rats from ischemic brain injury in vivo. By contrast, miR-101 inhibitor exacerbated growth defect, apoptosis, and ischemic brain injury. Luciferase reporter assay indicated that JAK2 was a direct target of mIR-101, and JAK2 silencing effectively reversed the miR-101 inhibitor-induced neuronal cell apoptosis in vitro and reduced cerebral infarction volume in vivo.

Conclusion: Our study demonstrated that miR-101 efficiently protected neuronal cells from apoptosis and ischemic brain injury through regulating the JAK2/STAT3 signaling pathway, suggesting that miR-101 might be a potential target for treatment of ischemic stroke.

Keywords: ischemic stroke, miR-101, JAK2/STAT3 signaling

\section{Introduction}

Stroke has become the fifth cause of death in the USA. ${ }^{1,2}$ Approximately $87 \%$ of strokes are ischemic, ${ }^{3}$ and ischemic stroke is always characterized by acute loss of neurons, astroglia, and oligodendroglia and disruption of synaptic architecture due to cerebral artery occlusion. ${ }^{4}$ Although several clinical trials have been conducted, there is no established treatment to reduce the neurological deficits caused by ischemic stroke. ${ }^{5,6}$ Many patients suffering from stroke often remain disabled after active therapies. ${ }^{7}$ Therefore, well understanding the specific mechanism in ischemic stroke will contribute to identifying and developing new therapeutic targets.

MicroRNAs (miRNAs), which are approximately 20 nucleotides in length and lack protein-coding capacity, can regulate many target genes at post-transcriptional level. ${ }^{8}$ Previous studies have reported that miRNAs play important roles in 
neuroprotection and post-stroke recovery. For instance, miR-148b inhibits proliferation and differentiation of neural stem cells through attenuating Wnt/ $\beta$-catenin pathway in mouse ischemic stroke model. ${ }^{9}$ MiR-9a-5p protects against ischemia injury through regulating ATG5-mediated autophagy. ${ }^{10}$ MiR-15a/16-1 antagomir attenuates ischemic brain injury in the experimental stroke. ${ }^{11}$ MiRNA-27b downregulation promotes neurogenesis through activating the AMPK signaling pathway. ${ }^{12}$ In addition, neural stem/ progenitor cells (NSPCs)-based treatment is a potential therapeutic approach for stroke. A series of miRNAs are considered promising biomarkers for diagnosis and prognosis and targets for controlling the proliferation, differentiation, and migration of NSPCs. ${ }^{13}$ It has been demonstrated that miR-101 is dramatically reduced in numerous human cancers and has potent anti-tumor effects through inhibiting cell proliferation and metastasis. ${ }^{14} \mathrm{MiR}-$ 101 inhibits cell proliferation and invasion of pancreatic cancer by directly targeting STMN1. ${ }^{15}$ MiR-101 also suppresses breast cancer cell proliferation and enhances their sensitivity to oxidative stress via $\mathrm{Nrf} 2$ signaling pathway. ${ }^{16}$ However, its role in neurogenesis after ischemic stroke remains unclear.

Studies have shown that JAK/STAT3 signaling pathway could regulate different biological processes in human cancer cells, including proliferation, differentiation, invasion, and migration. ${ }^{17}$ IL-6 secreted by cancer-associated fibroblasts could promote epithelial-mesenchymal transition and metastasis of gastric cancer via JAK2/STAT3 signaling pathway. ${ }^{18}$ LncRNA PICART1 suppresses proliferation and promotes apoptosis of lung cancer cells by inhibiting JAK2/STAT3 pathway. ${ }^{19}$ Increasing evidence indicated that JAK/STAT pathway plays a crucial role in regulating ischemic stroke-induced inflammatory neuronal damages. ${ }^{20}$ Constitutive activation of the JAK-STAT pathway has been observed in cerebral ischemia. ${ }^{21}$ All these indicate that JAK2/STAT3 signaling pathway has an important impact on ischemic stroke. Recently, miRNAs have been identified as major regulators of the JAK2/ STAT3 signaling pathway in cerebral ischemic injury. ${ }^{20}$ Therefore, our study focused on the mechanism underlying the interaction between miR-101 and JAK2/STAT3 pathway in ischemic stroke.

Here, our study first demonstrated that miR-101 was significantly downregulated in both brain tissues of rats with $\mathrm{I} / \mathrm{R}$ injury and OGD/R-induced neuronal cells. Further, our results identified that JAK2 was a target of miR-101 with two specific-binding sites. Moreover, our study revealed that miR-101 attenuated ischemic brain injury by inhibiting the JAK2/STAT3 signaling pathway, which provided a novel therapeutic target for ischemic stroke.

\section{Materials and Methods Cell Isolation and Culture}

Neuronal cells were isolated from the cerebral cortex of SD rats (approximately 17-days old) as previously described. ${ }^{22}$ Cells were cultured at $37{ }^{\circ} \mathrm{C}$ with $95 \%$ air and $5 \% \mathrm{CO}_{2}$ in DMEM medium supplemented with $10 \%$ FBS, 100 units $/ \mathrm{mL}$ penicillin, and $0.1 \mathrm{mg} / \mathrm{mL}$ streptomycin.

\section{OGD/R Model}

The neurons were exposed to $\mathrm{OGD} / \mathrm{R}$ as previously described. ${ }^{23}$ Briefly, neuronal cells were exposed to oxygen-glucose deprivation (OGD) for $2 \mathrm{~h}$ and transferred into DMEM media containing glucose under normal conditions for reoxygenation. Control cells were cultured in glucose-containing DMEM under normal conditions.

\section{MCAO/R Model}

Adult male Sprague Dawley rats (approximately 8-10 weeks and weighing $270 \pm 17 \mathrm{~g}$ ) were purchased from Shaanxi Provincial People's Hospital. All experiments were approved by the Ethics Committee of Shaanxi Provincial People's Hospital. Procedures operated in this research were completed in keeping with the standards set out in the Health Laboratory Animal Care and Use Guidelines of Shaanxi Provincial People's Hospital. The $\mathrm{MCAO} / \mathrm{R}$ model was constructed as previously described. ${ }^{24}$ Briefly, rats were anesthetized with $100 \mathrm{mg} /$ $\mathrm{kg}$ ketamine and $10 \mathrm{mg} / \mathrm{kg}$ xylazine. A piece of 6-0 monofilament nylon suture with its cusp slightly rounded by heat was plugged via the right internal carotid artery to the base of the middle cerebral artery. After $2 \mathrm{~h}$, blood flow was restored by removing the suture. Sham control animals were subjected to similar operations to expose the carotid arteries without occlusion of the middle cerebral artery. After $2 \mathrm{~h}$ of MCAO, rats were allowed to reperfusion for $24 \mathrm{~h}$. The mortality rate of the MCAO model rats is $10 \%$.

\section{Cell Transfection}

In specific experiments, neuronal cells were transfected with miR-101 mimics, inhibitor, and corresponding 
controls by Lipofectamine 2000 kit according to the manufacturer's instructions. After transfection for $48 \mathrm{~h}$, cells were subjected to OGD/R treatment and collected for the subsequent experiments.

\section{Cortical Injection}

Cortical injections were performed as previously reported. ${ }^{25}$ Briefly, lentiviral sh-JAK2 or NC control was mixed with cationic lipid polybrene for $15 \mathrm{~min}$ at $37^{\circ} \mathrm{C}$, and $7 \mu \mathrm{L}$ of the mixture was administered. $100 \mu \mathrm{M}$ miR101 mimics, miR-101 inhibitor or NC controls were mixed with the siRNA-Mate for 20 min and subjected to cortical injection. In short, rats were anesthetized with chloral hydrate $(10 \%)$ and placed in the stereotactic apparatus (anteroposterior $0.8 \mathrm{~mm}$, mediolateral $1.6 \mathrm{~mm}$, depth $3.5 \mathrm{~mm}$ ). After injection, rats were exposed to MCAO/R and used for the subsequent experiments.

\section{Measurement of Infarct Volume}

After $\mathrm{MCAO} / \mathrm{R}$, rats were sacrificed. The brains were quickly removed and cut into $1.0 \mathrm{~mm}$-thick coronal sections. The sections were incubated with $0.5 \%$ 2,3,5-triphenyl tetrazolium chloride (TTC) solution at $37^{\circ} \mathrm{C}$ for 15 min, observed, and scanned with a computer. The infarct volume shown in the images was analyzed using ImagePro Plus1 6.0 software.

\section{Animal Model Inclusion and Exclusion Criteria}

Following cerebral ischemia, rats were tested for neurological deficits and scored on a 5-point scale as previously described. ${ }^{26}$ Rats were excluded if the following occurred: (1) Anesthesia accident; (2) severe hemorrhage during the operation, (3) death or apparent surgical injury; (4) died before the sampling; (5) subarachnoid hemorrhage during the sampling; and (6) brain tissue infarction. The included rats were scored 0 point if no observable neurological deficits (normal), 1 point if failing to extend right forepaw (mild), 2 points if circling to the contralateral side (moderate), 3 points if falling down to the left side, and 4 points if unable to move by themselves and losing consciousness.

\section{RNA Extraction and qRT-PCR}

Total RNAs of the cultured cells or brain tissues were extracted using TRIzol reagent according to the manufacturer's instructions. Reverse transcription was performed using the M-MLV Reverse Transcriptase kit.
Quantitative real-time PCR was performed by a SYBR Green-based Rotor-Gene RG-3000A. The relative expression change of target genes was analyzed by the $2^{-\Delta \Delta \mathrm{Ct}}$ method with U6-snRNA and GAPDH as the internal references. The primers used in this study were miR-101 forward 5'-GTACAGTACTGTGATAACTGA $-3^{\prime}$ and reverse 5'-TGCGTGTCGTG GAGTC-3'; JAK2 forward 5'-GGGAGGTGGTCGCTGTAAAA- $3^{\prime}$ and reverse 5'-ACCAGCACT GTAGCACACTC-3'; U6 forward 5'-CTCGCTTCGGCAGCACA-3' and reverse 5'AACGCTTCACGAATTTGCGT-3'; and GAPDH forward 5'-CCTCG TCCCGTAGACAAAATG-3' and reverse 5'-TCTCCACTTTGCCACTGCAA-3'.

\section{Western Blot}

Total proteins of the cultured cells or brain tissues were extracted using RIRP lysis buffer. Approximate equal protein was separated by $10 \%$ SDS-PAGE and transferred onto a PVDF membrane. After blocking in TBS-Tween buffer containing $20 \mathrm{mM}$ Tris- $\mathrm{HCl}, 5 \%$ nonfat milk, $150 \mathrm{mM}$ $\mathrm{NaCl}$, and $0.05 \%$ Tween- $20(\mathrm{pH} 7.5)$, the membrane was incubated with 1:500 diluted primary antibodies against JAK2 (ab108596, Abcam), STAT3 (ab119352, Abcam), p-STAT3 (ab30647, Abcam) or GAPDH (1:1000, ab9485, Abcam) overnight at $4^{\circ} \mathrm{C}$. Finally, the membrane was exposed to secondary antibodies labeled with horseradish peroxidase (HRP) (1:5000, ab6721, Abcam) for $1 \mathrm{~h}$. The target proteins were detected using the enhanced chemiluminescence kit and analyzed using the Blot AP System.

\section{Luciferase Reporter Assay}

The luciferase reporter plasmid containing wild-type (WT) JAK2-3'-UTR and mutant (MUT) JAK2-3'-UTR were constructed and co-transfected with miR-101 mimic or NC control into neuronal cells using Lipofectamine 2000. After transfection for $48 \mathrm{~h}$, cells were collected, and the Renilla luciferase activities were detected by the dual-luciferase assay system.

\section{MTT Assay}

Cell viability was detected using MTT Cell Viability Assay Kit. Briefly, cells were seeded into 96-well plates and cultured overnight. After that, $10 \mu \mathrm{L}$ of MTT stock solution was added to each well. Cells were incubated at $37{ }^{\circ} \mathrm{C}$ for 4 h. The formed crystal substances were dissolved in $100 \mu \mathrm{L}$ of dissolution reagent, and the absorbance was measured at $570 \mathrm{~nm}$ using a microplate absorbance reader. 


\section{Flow Cytometry Analysis}

Cell apoptosis was quantified by flow cytometry analysis (BD Biosciences, San Jose, CA, USA). Briefly, cells were trypsinized and resuspended in PBS. Then, $100 \mu \mathrm{L}$ of $1 \mathrm{x}$ binding buffer was added to each sample, followed by adding $5 \mu \mathrm{L}$ Annexin V fluorescein isothiocyanate (Annexin V-FITC, BD Biosciences) and $5 \mu \mathrm{L}$ propidium iodide (BDBiosciences). The samples were incubated in the dark for $20 \mathrm{~min}$ and subjected to flow cytometry after washed three times with PBS. The apoptotic rate was detected using the FACS Calibur flow cytometry and quantified by FlowJo software.

\section{Statistical Analysis}

SPSS software (version 18.0) was used for statistical analysis. All experiments were repeated at least three times, and data are presented as means $\pm \mathrm{SD}$. The difference between two groups was determined by Student's $t$-test. $\mathrm{P}<0.05$ was considered as the significant threshold.

\section{Results}

\section{miR-I0I Was Significantly \\ Downregulated After I/R Injury}

To explore the effects of miR-101 on ischemic brain damage, neuronal cells were subjected to OGD/R. The results indicated that neuronal cells subjected to $\mathrm{OGD} / \mathrm{R}$ showed a significantly reduced cell growth $(\mathrm{p}<0.01)$ (Figure 1A), increased apoptotic rate $(\mathrm{p}<0.01)$ (Figure $1 \mathrm{~B})$ and decreased miR-101 expression $(p<0.01)$ (Figure 1C). Furthermore, rats subjected to $\mathrm{MCAO} / \mathrm{R}$ also exhibited obviously increased neurological scores $(p<0.01)$ (Figure 1D) and infarct volume $(\mathrm{p}<0.01)$ (Figure $1 \mathrm{E})$, suggesting that $\mathrm{MCAO} / \mathrm{R}$ could lead to neurologic dysfunction and severe cerebral infarction. Meanwhile, miR-101 was significantly decreased in brain tissues $(\mathrm{p}<0.01)$ (Figure $1 \mathrm{~F})$ and plasma $(\mathrm{p}<0.01)$ (Figure $1 \mathrm{G}$ ) of rats with $\mathrm{I} / \mathrm{R}$ injury compared with the sham group. These results indicated that miR-101 was closely associated with cerebral I/R injury.
A

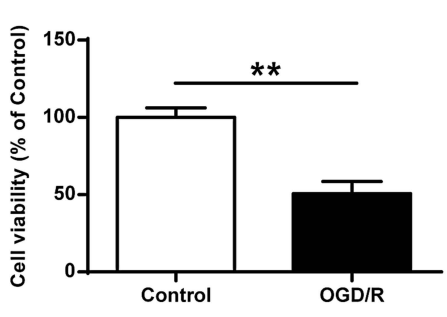

C

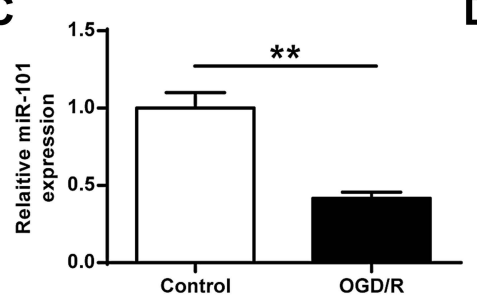

B

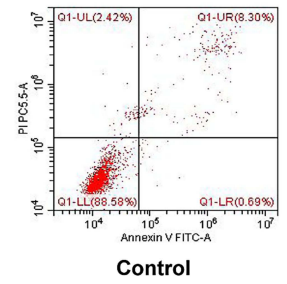

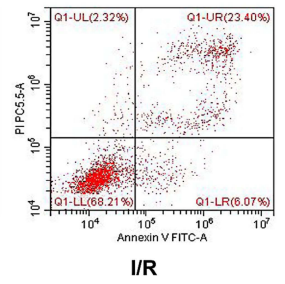

E
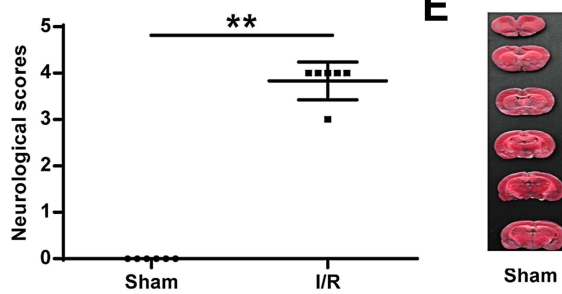
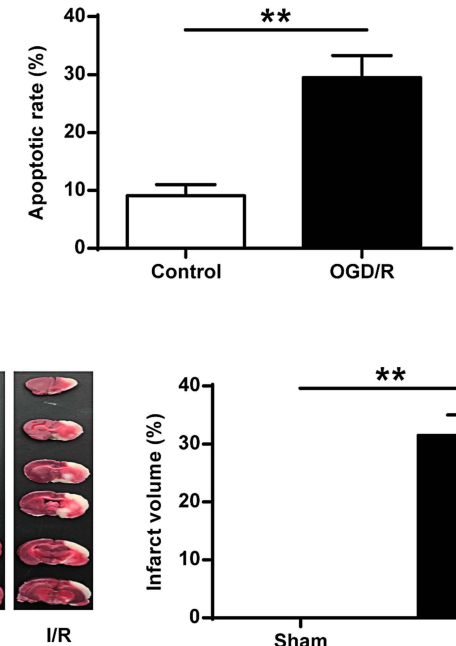

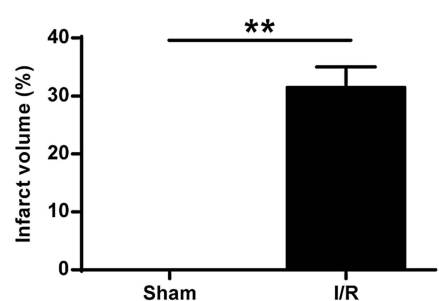

$\mathbf{F}$
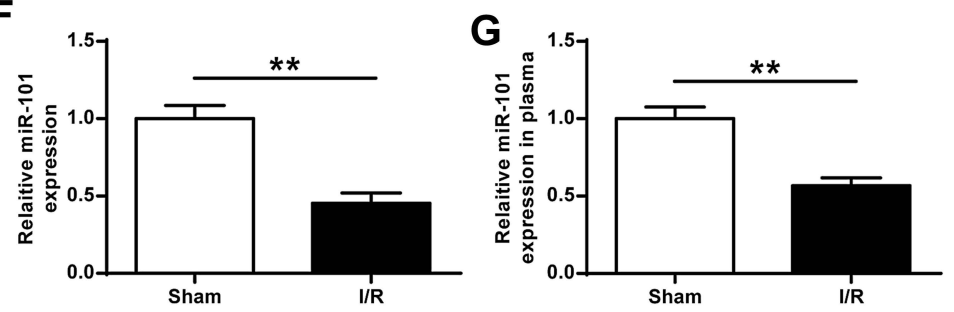

Figure I MiR-IOI was significantly downregulated after I/R in vitro or in vivo. (A-C) The neuronal cells were exposed to OGD/R. (A) The cell viability was measured by MTT assay $(n=3)$. (B) The apoptotic rate was detected by flow cytometry $(n=3)$. (C) The mRNA level of miR-I0I was evaluated by qRT-PCR $(n=3)$. $(\mathbf{D}-\mathbf{G})$ Rats were exposed to MCAO/R. (D) Neurobehavioral outcomes $(n=6)$. (E) Infarct volumes $(n=6)$. $(\mathbf{F}$ and $\mathbf{G})$ The mRNA level of miR-I0I in brain tissues $(\mathbf{F})$ and plasma $(\mathbf{G})$ was evaluated by qRT-PCR $(n=6)$. Data were presented as mean \pm SD. ${ }^{*} p<0.01$. 


\section{MiR-I0I Overexpression Protected} Neurons Against OGD/R-Induced Injury in vitro

Next, miR-101 mimics or inhibitor were transfected into neuronal cells, and the cell viability and apoptotic rate were evaluated. We firstly detected the transfection efficiency of miR-101 mimics and inhibitor, and the results showed that miR-101 mimics significantly increased miR101 expression and miR-101 inhibitor decreased miR-101 expression $(\mathrm{p}<0.01)$ (Figure 2A). Moreover, miR-101 mimics significantly attenuated OGD/R-induced growth defect in neuronal cells $(\mathrm{p}<0.01)$ while miR-101 inhibitor exacerbated OGD/R-induced neuronal cell death $(\mathrm{p}<0.05)$ (Figure 2B). Furthermore, OGD/R-induced neuronal cell apoptosis was reversed by miR-101 mimics $(\mathrm{p}<0.01)$ and promoted remarkedly by miR-101 inhibitor $(\mathrm{p}<0.05)$ (Figure 2C). These data indicated that miR-101 overexpression efficiently protected neurons against OGD/ R-induced injury in vitro.

\section{MiR-IOI Overexpression Attenuated Ischemic Brain Injury in vivo}

To confirm the protective role of miR-101 in cerebral I/R injury, miR-101 mimics or inhibitor were subcutaneously injected into the cerebral cortex of rats with $\mathrm{I} / \mathrm{R}$ injury. QRT-PCR results showed that miR-101 mimics significantly increased miR-101 expression $(\mathrm{p}<0.01)$ and miR101 inhibitor decreased its expression in vivo $(\mathrm{p}<0.01)$
A

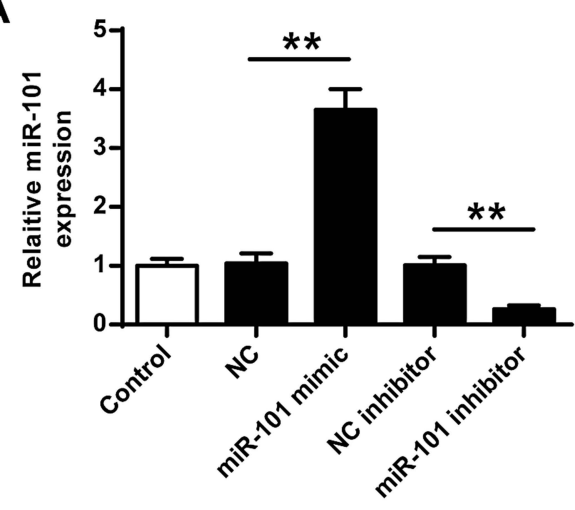

C
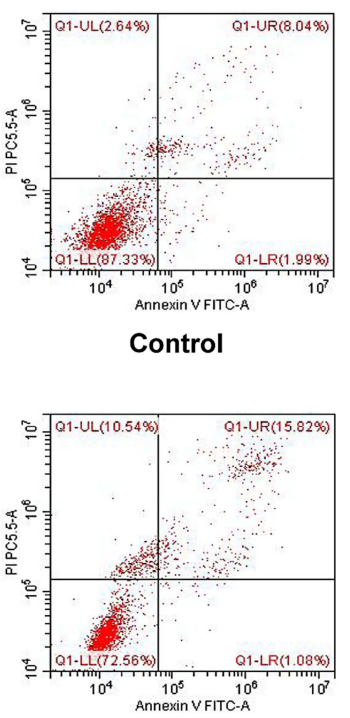

OGD/R+miR-101 mimic
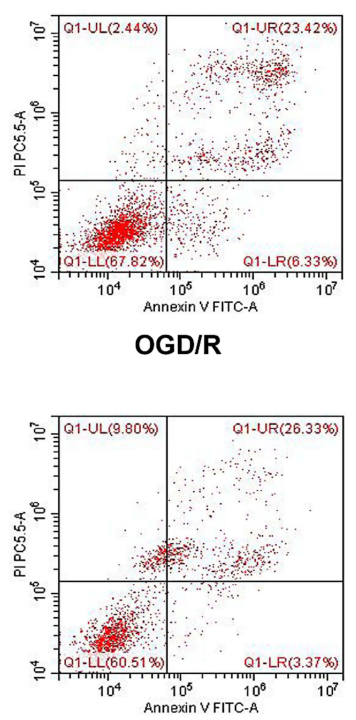

OGD/R+NC inhibitor
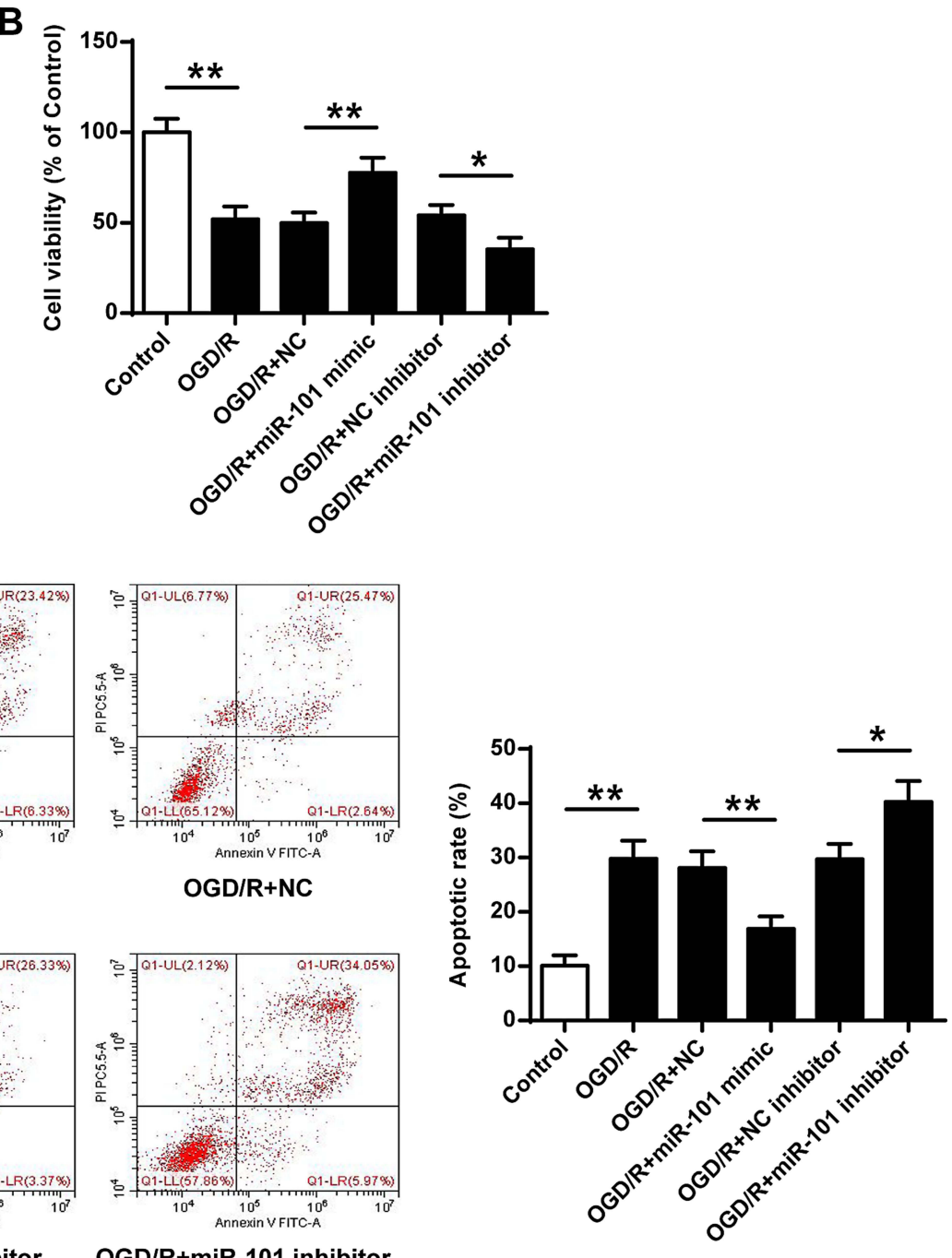

Figure 2 MiR-IOI upregulation protected neuronal cells against OGD/R-induced injury in vitro. (A) The neuronal cells were transfected with miR-I0I mimics, miR-NC, miR-IOI inhibitor, or NC inhibitor. The transfection efficiency was evaluated by qRT-PCR. (B and C) The neuronal cells were transfected with miR-I0I mimics, miR-NC, miR-IOI inhibitor, or NC inhibitor. Then cells were exposed to OGD/R. (C) Cell viability was evaluated by MTT assay. (C) The apoptosis was detected by flow cytometry. Data were presented as mean $\pm \mathrm{SD}$. $\mathrm{N}=3$. ${ }^{*} \mathrm{p}<0.05$, **p $<0.01$. 
(Figure 3A). MiR-101 mimics remarkedly decreased I/ $\mathrm{R}$-induced neurological scores in rats $(\mathrm{p}<0.01)$ (Figure 3B). Meanwhile, the infarct volume of rats with I/R injury was significantly reduced by miR-101 mimics $(p<0.01)$ and obviously exacerbated by miR-101 inhibitor $(\mathrm{p}<0.05)$ (Figure $3 \mathrm{C})$. These results confirmed the protective effect of miR-101 in ischemic brain injury.

\section{JAK2 Was a Direct Target of miR-I0I}

To explore how miR-101 exerts its protective effect in ischemic brain injury, miRNA database microRNA.org (http://www.microrna.org/microrna/home.do) was used to predict the potential targets of miR-101. The results indicated that JAK2 had two potential interactional sites with miR-101 (Figure 4A). Then, the luciferase reporter plasmids containing wild-type 3'-UTR of JAK2 (WT) or mutant-type 3'-UTR of JAK2 (MUT) were constructed and co-transfected with miR-101 mimics or miR-NC into neuronal cells. The luciferase reporter assay indicated that miR-101 mimics significantly inhibited the expression of WT 3'-UTR of JAK2 and exhibited no obvious change in MUT $3^{\prime}$-UTR of JAK2 both in site 1 and site $2(\mathrm{p}<0.01)$ (Figure 4B). Moreover, JAK2 mRNA level was significantly upregulated in OGD/ R-induced neuronal cells $(\mathrm{p}<0.01)$. In addition, JAK2 mRNA expression was inhibited by miR-101 mimics $(\mathrm{p}<0.01)$ and promoted by miR-101 inhibitor $(\mathrm{p}<$ 0.05) (Figure 4C). Similarly, JAK2 protein level was significantly upregulated in OGD/R-induced neuronal cells ( $\mathrm{p}<0.01$ ). MiR-101 mimics downregulated JAK2 protein level $(\mathrm{p}<0.01)$, and miR-101 inhibitor remarkedly increased JAK2 expression ( $\mathrm{p}<0.05$ ) (Figure 4D). These results indicated that JAK2 was a target of miR-101.
A

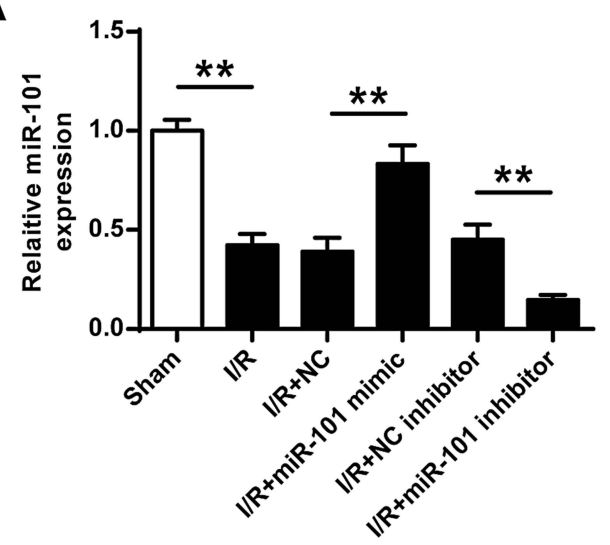

C
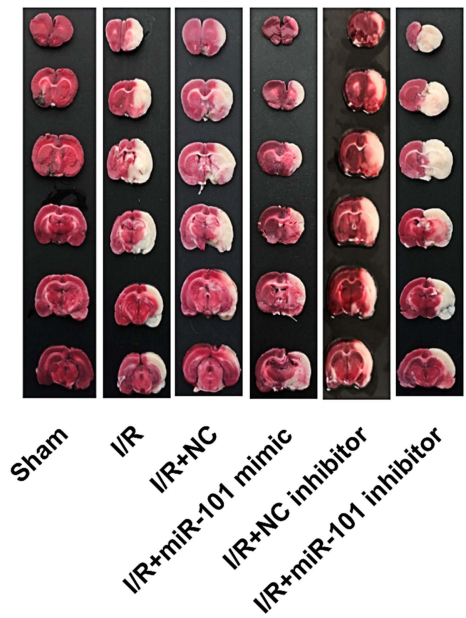

B
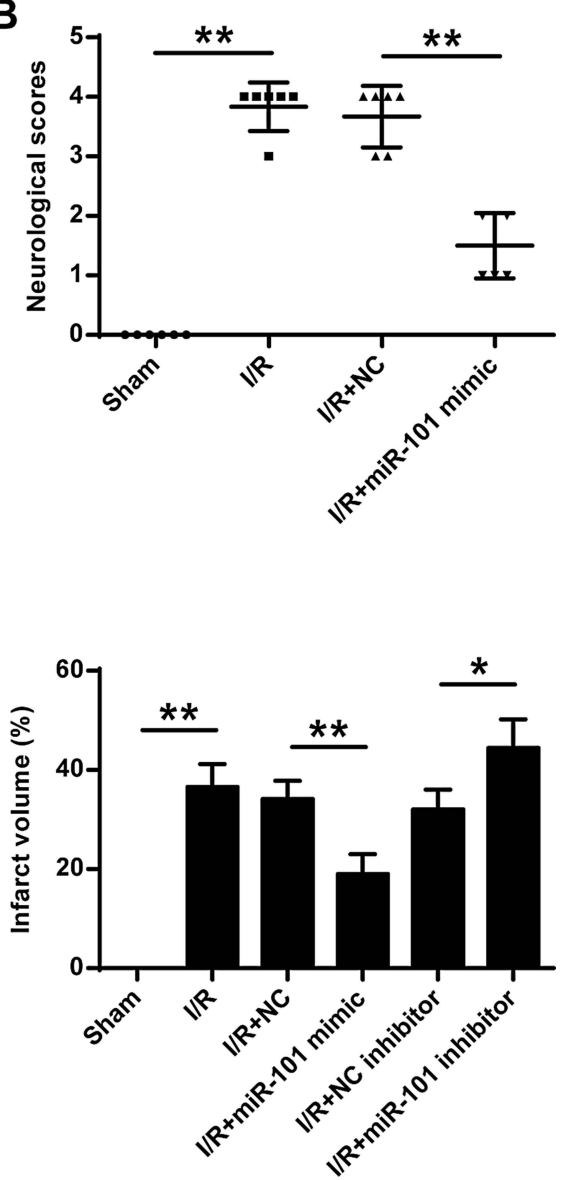

Figure 3 MiR-IOI upregulation attenuated ischemic brain injury in vivo. MiR-IOI mimics, miR-NC, miR-I0I inhibitor, or NC inhibitor were subjected to cortical injection, and rats were then exposed to MCAO/R. (A) MiR-I0I mRNA level was evaluated by qRT-PCR. (B) Neurobehavioral outcomes. (C) Infarct volumes. Data were presented as mean \pm SD. $N=6$. ${ }^{*} p<0.05$, **p $<0.01$. 


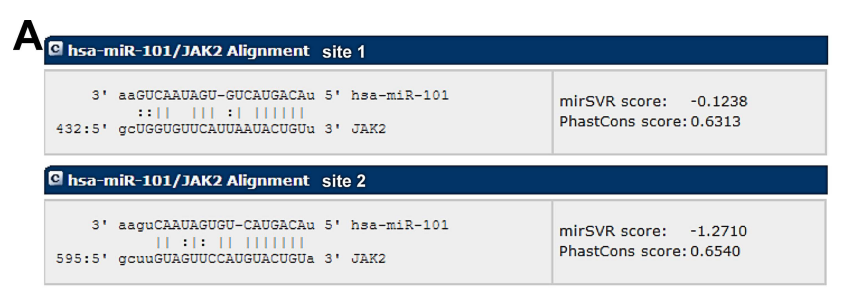

B
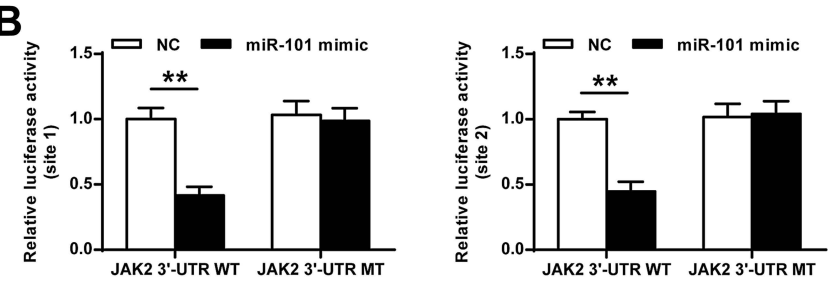

C
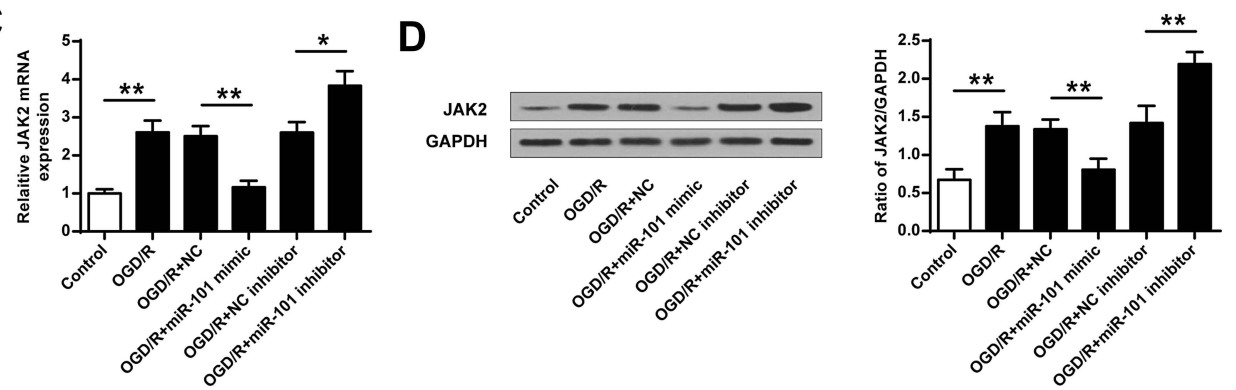

Figure 4 JAK2 was a target of miR-I0I. (A) The interactional sites between miR-I0I and JAK2. (B) The luciferase activities were detected by the dual-luciferase assay system. (C and D) The neuronal cells were transfected with miR-I0I mimics, miR-NC, miR-I0I inhibitor, or NC inhibitor. Then cells were exposed to OGD/R. The mRNA level (C) and protein level (D) of JAK2 were evaluated. Data were presented as mean \pm SD. $N=3$, *p $<0.05$, **p $<0.01$.

\section{JAK2 Downregulation Suppressed Neuronal Cell Apoptosis and Ischemic Brain Injury}

To further explore whether miR-101 could promote ischemic brain injury through JAK2, sh-JAK2 (silencing of JAK2), or sh-NC control were transfected into neuronal cells or subcutaneously injected into the cerebral cortex of rats with $\mathrm{I} / \mathrm{R}$ injury. In vivo, sh-JAK2 significantly decreased JAK2 at both mRNA $(\mathrm{p}<0.01)$ (Figure 5A) and protein levels $(\mathrm{p}<0.01)$ (Figure $5 \mathrm{~B})$ compared with sh-NC. The phosphorylation level of STAT3 was significantly increased in OGD/R-induced neuronal cells $(\mathrm{p}<$ 0.01 ), and sh-JAK2 dramatically decreased the phosphorylation level of STAT3 compared with sh-NC $(\mathrm{p}<0.01)$ (Figure 5C). Sh-JAK2 could attenuate OGD/R-induced growth defect $(\mathrm{p}<0.05)$ (Figure 5D) and inhibit OGD/ R-induced apoptosis $(\mathrm{p}<0.05)$ (Figure $5 \mathrm{E})$ in neuronal cells compared with sh-NC. In vitro, JAK2 expression was remarkedly upregulated in the ischemic brain, and shJAK2 efficiently downregulated JAK2 expression in both mRNA $(\mathrm{p}<0.01)$ (Figure 5F) and protein $(\mathrm{p}<0.01)$ levels (Figure $5 \mathrm{G}$ ) in the ischemic brain compared with sh-NC.
Moreover, sh-JAK2 efficiently decreased the neurological scores $(\mathrm{p}<0.01)$ (Figure $5 \mathrm{H})$ and infarct volume $(\mathrm{p}<$ 0.05 ) (Figure 5I). These data indicated that sh-JAK2 suppressed neuronal cell apoptosis in vitro and protected against ischemic brain injury in vivo.

\section{MiR-IOI Protected Ischemic Brain Injury by Targeting JAK2}

To confirm whether miR-101 inhibited ischemic brain injury by regulating JAK2 expression, the OGD/ R-induced neuronal cells were co-transfected with miR101 mimics and sh-JAK2, or miR-101 inhibitor and shJAK2. The results indicated that additional sh-JAK2 significantly downregulated JAK2 expression compared with miR-mimics group $(\mathrm{p}<0.05)$, and co-transfection of miR-101 inhibitor and sh-JAK2 obviously attenuated miR-101 inhibitor-promoted JAK2 expression $(\mathrm{p}<0.05)$ (Figure 6A). Meanwhile, co-transfection of miR-101 mimics and sh-JAK2 significantly downregulated p-STAT3 level compared with miR-101 mimics group $(\mathrm{p}<0.05)$, while co-transfection of miR-101 inhibitor and sh-JAK2 obviously attenuated miR-101 inhibitorpromoted p-STAT3 expression compared with miR-101 
A

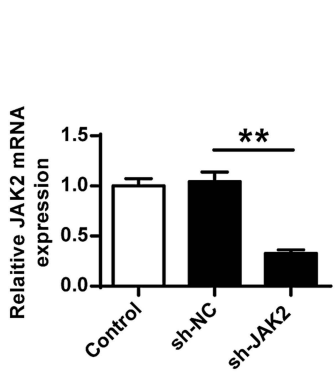

E

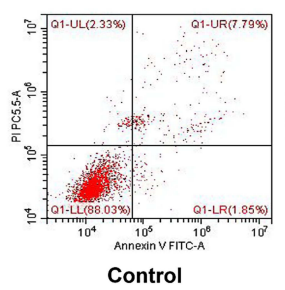

F

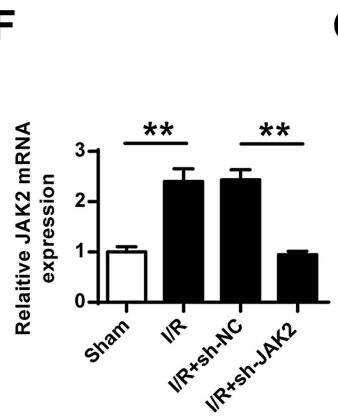

B

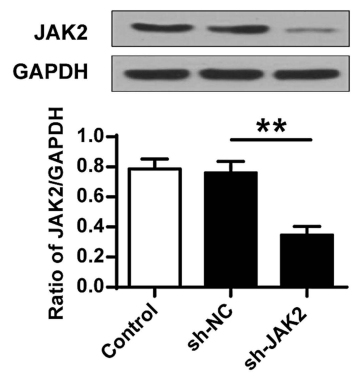

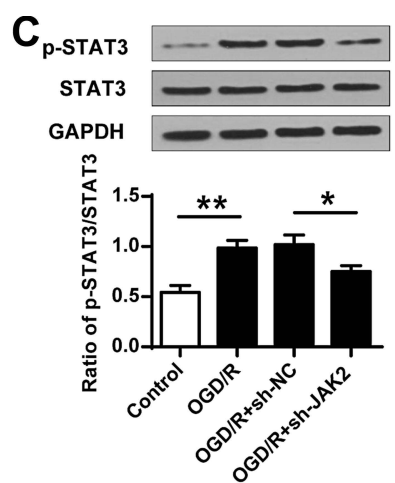
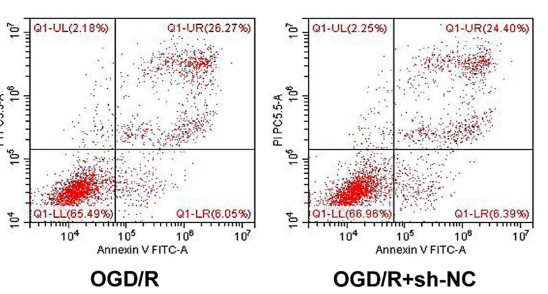

OGD/R+sh-NC

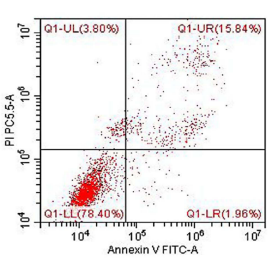

OGD/R+sh-JAK2
D
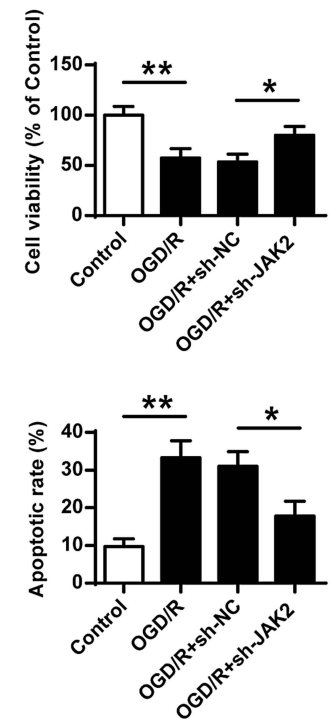

$\mathbf{G}_{\text {JAK2 }}$

H

I
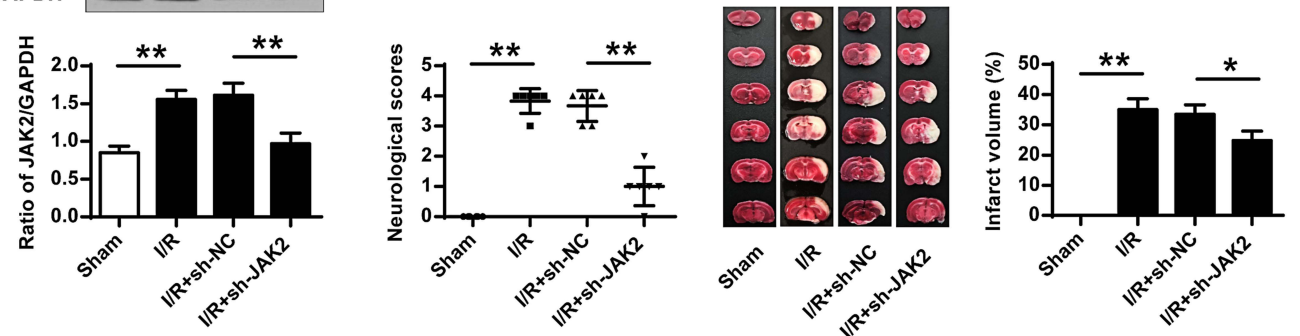

Figure 5 Sh-JAK2 suppressed neuronal cell death and ischemic brain injury. (A-E) The neuronal cells were transfected with sh-JAK2 or sh-NC, and cells were exposed to OGD/R or not. (A) JAK2 mRNA level was evaluated by qRT-PCR $(n=3)$. (B and $\mathbf{C})$ The protein levels of JAK2 (B) and p-STAT3 (C) were evaluated by Western blot $(n=3)$. (D) Cell viability was detected by MTT assay $(n=3)$. (E) The apoptosis was detected by flow cytometry $(n=3)$. $(\mathbf{F}-\mathbf{I})$ Cortical injection of sh-JAK2 or sh-NC, and rats were then exposed to MCAO/R or not. $(\mathbf{F})$ JAK2 mRNA level was evaluated by qRT-PCR $(n=6)$. $(\mathbf{G})$ JAK2 protein level was evaluated by Western blot $(n=6)$. (H) Neurobehavioral outcomes $(n=6)$. (I) Infarct volumes $(n=6)$. Data were presented as mean \pm SD. $*_{p}<0.05$, **p $<0.0$ l.

inhibitor $(\mathrm{p}<0.05)$ (Figure 6B). Moreover, additional sh-JAK2 promoted neuronal cell growth compared with miR-101 mimics $(p<0.05)$ and protected miR-101 inhibitor-caused growth defect in OGD/R-induced neuronal cells $(\mathrm{p}<0.05)$ (Figure 6C). For apoptosis, additional shJAK2 significantly inhibited neuronal cell apoptosis compared with miR-101 mimics group $(\mathrm{p}<0.05)$ while additional sh-JAK2 inhibited miR-101 inhibitor-induced apoptosis $(\mathrm{p}<0.05)$ (Figure 6D). Further, additional shJAK2 more effectively decreased the infarct volume caused by miR-101 mimics $(\mathrm{p}<0.05)$, and protected against miR-101 inhibitor- exacerbated cerebral infarction volume $(\mathrm{p}<0.05)$ (Figure $6 \mathrm{E})$. These data demonstrated that miR-101 inhibited ischemic brain injury partly by suppressing JAK2 expression.

\section{Discussion}

Ischemic stroke is a common injury-caused neurological disease with a higher mortality and disability rates. ${ }^{27}$ Cognitive dysfunction often follows a stroke, and a clinical report has suggested it occurs in $50-75 \%$ of patients. ${ }^{28}$ Although the survival rate of patients has been improved with the development of medical technology, the disability rate has still increased accordingly. ${ }^{29}$ Hence, the identification of novel therapeutic targets in ischemic stroke is more urgent and can help clinicians to develop efficient therapeutic strategies.

Neuronal cell death is considered a critical part of stroke pathophysiology, and a variety of miRNAs have been identified to exert their roles in the progression of ischemic injury by inducing brain cell death. MicroRNAs 
A
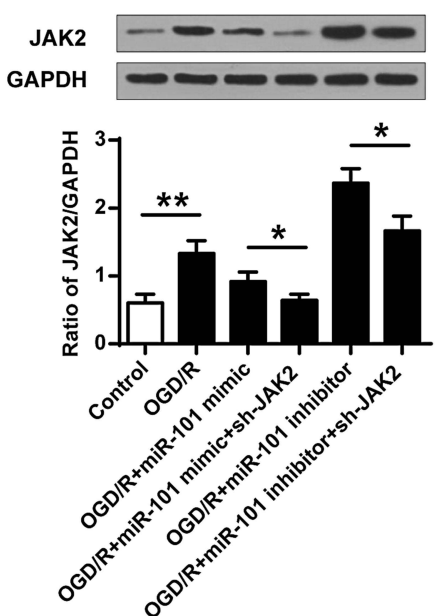

B

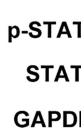

P-STAT3
STAT3
GAPDH

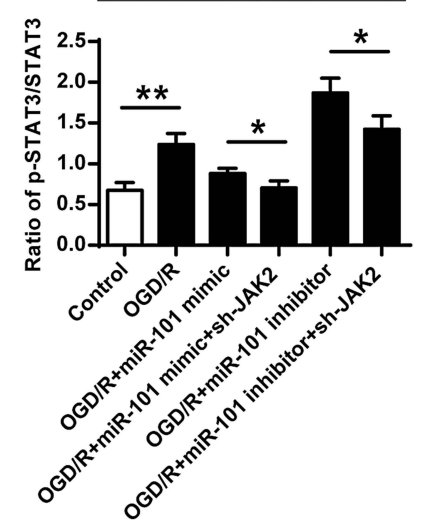

C

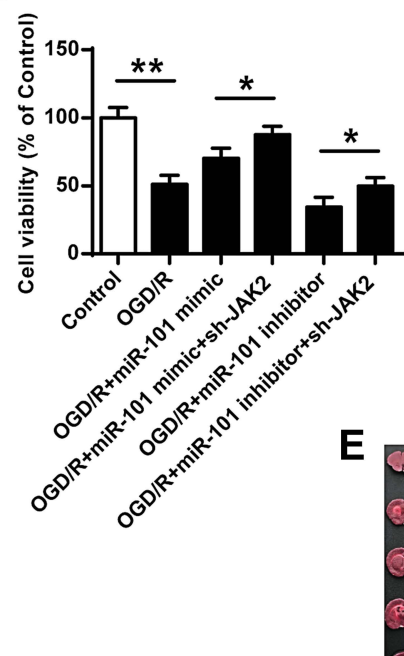

D

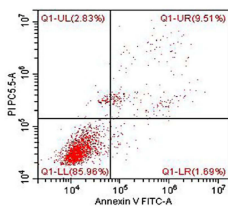

Control

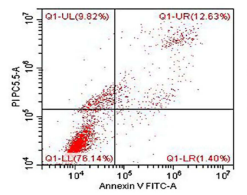

OGD/R+miR-101 mimic +sh-JAK2

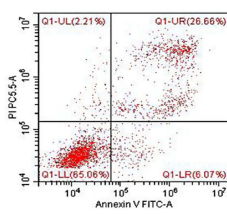

OGD/R

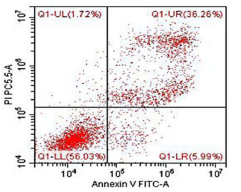

OGD/R+miR-101 inhibitor

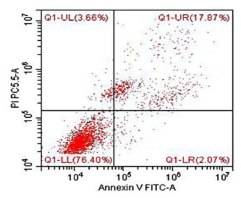

OGD/R+miR-101 mimic

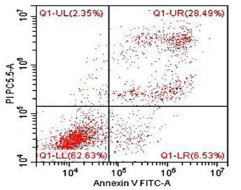

OGD/R+miR-101 inhibitor+sh-JAK2
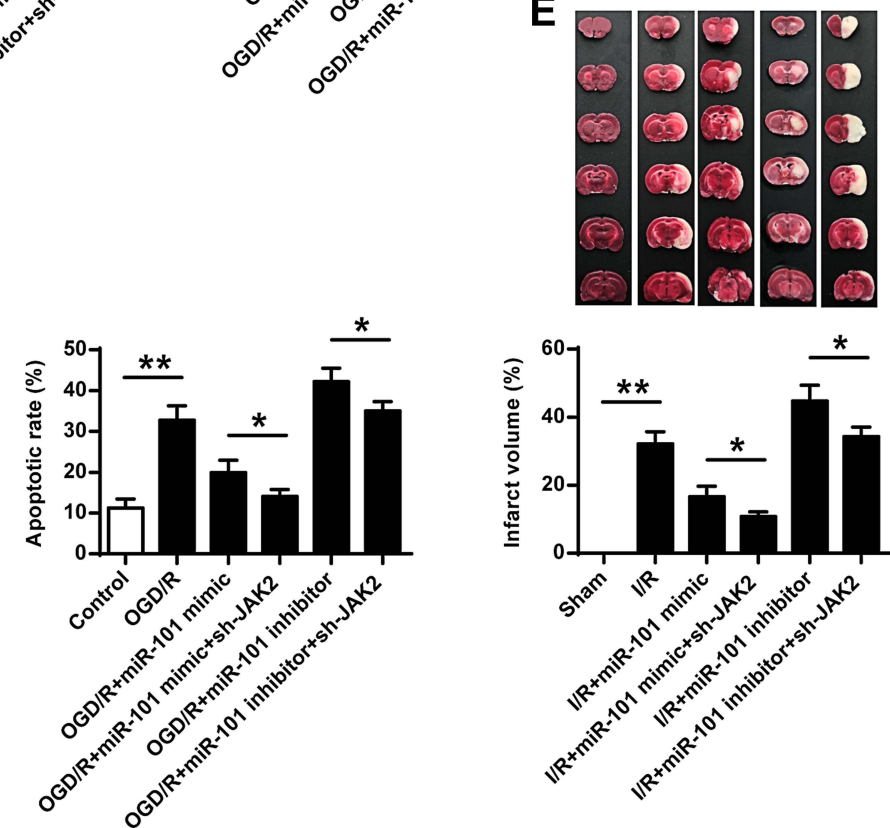

Figure 6 MiR-10I protected ischemic brain injury through targeting JAK2. (A-D) The neuronal cells were transfected with miR-I0I mimics or miR-I0I inhibitor, or cotransfected with miR-I0I mimics and sh-JAK2, or miR-I0I inhibitor and sh-JAK2, then cells were exposed to OGD/R or not. (A and B) The protein levels of JAK2 (A) and P-STAT3 $(\mathbf{B})$ were evaluated by Western blot $(n=3)$. (C) The cell viability was evaluated by MTT assay $(n=3)$. (D) The apoptosis was detected by flow cytometry $(n=3)$. (E) Rats were subjected to cortical injection of miR-I0I mimics, miR-I0I inhibitor, or cortical co-injection with miR-I0I mimics and sh-JAK2, or miR-I0I inhibitor and shJAK2, and were then exposed to MCAO/R. Infarct volume in rats with cerebral I/R injury $(n=6)$. Data were presented as mean \pm SD. *p $<0.05$, ** $p<0.01$.

as critical regulators of gene expression are important for functions including neuronal development, synapse formation. MiR-101 is a highly abundant miRNA in the brain tissue. The regulatory function of miR-101 mainly involves brain development, neuronal differentiation, neurogenesis regulation, glial cell and astrocyte differentiation, and other biological processes. MiR-101 has been identified to play an important anti-tumor role in various human cancers. MiR-101 overexpression augments cytotoxicity and reduces chemoresistance to chemotherapeutic reagent cisplatin (CDDP) in liver cancer cell line HepG2 cells through inhibiting the DNA-PKcs/Akt/NF- $\kappa \mathrm{B}$ signaling pathway. ${ }^{30}$ Circular RNA circVAPA is upregulated and exerts oncogenic properties by sponging miR-101 in colorectal cancer. ${ }^{31}$ MiR-101 has been reported to inhibit Nrf2 expression from impeding breast cancer cell proliferation and enhancing their sensitivity to oxidative stress. ${ }^{16}$ Meanwhile, abnormal miR-101 expression can be considered as potential diagnostic and prognostic biomarkers in different malignancies, including bladder cancer, mantle cell lymphoma, gastric cancer, and cervical cancer. ${ }^{32-35}$ In addition, miR-101 is also regarded as an efficient medicinal target to act its function. Berberine (BBR) can suppress endometrial cancer cell growth and metastasis through regulating miR-101/COX-2 axis. ${ }^{36}$ Studies have shown that miR-101 regulates hippocampal neuronal substance expression and amylin metabolism. ${ }^{37}$ However, the role of miR-101 in ischemic stroke has not been reported. In this study, we demonstrated that miR-101 was significantly downregulated in brain tissues of rats with $I / R$ 
injury and OGD/R-induced neuronal cells, suggesting its protective effect in ischemic stroke. To determine its role, miR-101 overexpression and inhibition assays were performed. The results indicated that miR-101 upregulation protected against cerebral I/R injury, and miR-101 downregulation exacerbated cerebral $\mathrm{I} / \mathrm{R}$ injury both in vitro and in vivo. These results confirmed the protective effect of miR-101 in ischemic stroke.

As known, under cerebral ischemic conditions, activation of JAK2 and STAT3 pathway contributes to neuronal damage. ${ }^{38}$ Moreover, the neuroprotective effects of the JAKSTAT signaling pathway inhibitor have also been identified and developed in the treatment of focal cerebral ischemia/ reperfusion injury in rat model. ${ }^{38}$ Atractylenolide III (A III), a sesquiterpene lactone, could reduce complications associated with ischemia partly through JAK2/STAT3-dependent mitochondrial fission in microglia. ${ }^{40}$ S14G-humanin (HNG), generated by replacement of Ser14 with glycine, exerts neuroprotective effects against $\mathrm{OGD} / \mathrm{R}$ by reactivating through JAK2/STAT3 signaling pathway. ${ }^{41}$ In this study, we found that JAK2 downregulation inhibits neuronal cell apoptosis and ischemic brain injury, confirming that JAK2/STAT3 pathway is an efficient drug target for ischemic stroke. Moreover, in ischemic stroke, miRNAs have been identified to act crucial regulatory functions through directly targeting JAK2/STAT3 signaling pathway. miR-216a overexpression significantly induced neuroprotection against ischemic injury through targeting JAK $2 .{ }^{20}$ In addition, JAK2/STAT3 pathway was also regulated by ncRNAs in many human cancers. LncRNA SNHG16 has been identified to act as an oncogene by directly sponging miR-135a and activating the JAK2/ STAT3 pathway in gastric cancer. ${ }^{42}$ LncRNA PICART1 can inhibit cell proliferation and promote apoptosis in lung cancer cells via inhibiting JAK2/STAT3 pathway. ${ }^{19}$ miRNA-133b and miRNA-135a have been reported to promote apoptosis by activating the JAK2/STAT3 pathway in renal carcinoma. ${ }^{43}$ MicroRNA-146a can promote the growth of acute leukemia cells by downregulating the expression of ciliary neurotrophic factor receptor and activating the JAK2/STAT3 signaling pathway. ${ }^{44}$ Studies have shown that miR101 could affect cell growth in rats with hepatopulmonary syndrome by inhibiting the JAK2/STAT3 pathway. Here, our results identified that JAK2 was a target of miR-101 with two binding sites. The luciferase reporter assay confirmed that miR-101 mimics could remarkedly decrease the expression of WT $3^{\prime}$-UTR of JAK2, while showed no obvious impact on MUT 3'-UTR of JAK2, confirming that miR-101 negatively regulates JAK2 by directly targeting 3'-UTR of JAK2.
Further, we found that miR-101 mimics also inhibited p-STAT3 level and miR-101 inhibitor increased p-STAT3 level. Moreover, additional sh-JAK2 efficiently attenuated miR-101 inhibitor exacerbated ischemic injury and enhanced the protective effect of miR-101 mimic. All these indicate that MiR-101 might protect brain I/R damage by regulating the JAK2/STAT3 signaling pathway. However, the exact relationship between miR-101 and cerebral infarction needs to be investigated further. Because the effect of miR-101 might provide a novel therapeutic approach for the treatment of ischemic injury, further studies may pave the way for the clinical usage of miR-101.

\section{Conclusion}

Our results demonstrated that miR-101 exerts a protective role in ischemic brain injury. Specifically, miR-101 prevented ischemic brain injury by targeting JAK2 and then inhibiting the STAT3 pathway. Our study suggested that miR-101/JAK2 might be a potential therapeutic target.

\section{Abbreviations}

$\mathrm{I} / \mathrm{R}$, Ischemia-reperfusion; OGD/R, Oxygen glucose deprivation-reoxygenation; $\mathrm{MCAO} / \mathrm{R}$, middle cerebral artery occlusion-reperfusion; TTC, Triphenyltetrazolium chloride; JAK2, Janus kinase 2; STAT3, signal transducer and activator of transcription 3; ceRNA, competing endogenous RNA; MTT, Methyl thiazolyl tetrazolium.

\section{Availability of Supporting Data}

The data are not publicly available due to their containing information that could compromise the privacy of research participants. However, they are available on request from the corresponding author: Zhijun Yong, Department of Rehabilitation Medicine, Shaanxi Provincial People's Hospital, No. 256 West Friendship (Youyi) Road, Xi'an City, Shaanxi Province, 710068, P. R. China. Email: zhijunyongmedicine@163.com.

\section{Ethical Approval and Consent to Participate}

All experiments were approved by the Ethics Committee of Shaanxi Provincial People's Hospital. Procedures operated in this research were completed in keeping with the standards set out in the Health Laboratory Animal Care and Use Guidelines of Shaanxi Provincial People's Hospital. 


\section{Disclosure}

The authors report no conflicts of interest in this work.

\section{References}

1. Murray CJ, Vos T, Lozano R, et al. Disability-adjusted life years (DALYs) for 291 diseases and injuries in 21 regions, 1990-2010: a systematic analysis for the Global Burden of Disease Study 2010. Lancet. 2012;380(9859):2197-2223. doi:10.1016/S0140-6736(12)61689-4

2. Mozaffarian D, Benjamin EJ, Go AS, et al. Heart disease and stroke statistics-2015 update: a report from the American Heart Association. Circulation. 2015;131(4):e29-322.

3. Fluri F, Schuhmann MK, Kleinschnitz C. Animal models of ischemic stroke and their application in clinical research. Drug Des Devel Ther. 2015;9:3445-3454.

4. Hao L, Zou Z, Tian H, Zhang Y, Zhou H, Liu L. Stem cell-based therapies for ischemic stroke. Biomed Res Int. 2014;2014:468748. doi: $10.1155 / 2014 / 468748$

5. Marder VJ, Stewart D. Towards safer thrombolytic therapy. Semin Hematol. 2002;39(3):206-216. doi:10.1053/shem.2002.34088

6. Malhotra K, Rayi A, Khunger M, Thompson S, Liebeskind DS. Reporting compliance of stroke trials: cross-sectional analysis. J Stroke Cerebrovasc Dis. 2017;26(7):1472-1480. doi:10.1016/j. jstrokecerebrovasdis.2017.03.010

7. Sharobeam A, Jones B, Walton-Sonda D, Lueck CJ. Factors delaying intravenous thrombolytic therapy in acute ischaemic stroke: a systematic review of the literature. $J$ Neurol. 2020:268(8):27232734. doi:10.1007/s00415-020-09803-6

8. Bartel DP. MicroRNAs: genomics, biogenesis, mechanism, and function. Cell. 2004;116(2):281-297. doi:10.1016/S0092-8674(04) 00045-5

9. Wang J, Chen T, Shan G. miR-148b regulates proliferation and differentiation of neural stem cells via $\mathrm{Wnt} / \beta$-catenin signaling in rat ischemic stroke model. Front Cell Neurosci. 2017;11:329. doi:10.3389/fncel.2017.00329

10. Wang N, Yang L, Zhang H, et al. MicroRNA-9a-5p alleviates ischemia injury after focal cerebral ischemia of the rat by targeting ATG5-mediated autophagy. Cell Physiol Biochem. 2018;45 (1):78-87. doi:10.1159/000486224

11. Yang X, Tang X, Sun $P$, et al. MicroRNA-15a/16-1 antagomir ameliorates ischemic brain injury in experimental stroke. Stroke. 2017;48 (7):1941-1947. doi:10.1161/STROKEAHA.117.017284

12. Wang Z, Yuan Y, Zhang Z, Ding K. Inhibition of miRNA-27b enhances neurogenesis via AMPK activation in a mouse ischemic stroke model. FEBS Open Bio. 2019;9(5):859-869. doi:10.1002/ 2211-5463.12614

13. Zhang Q, Zhang K, Zhang C, et al. MicroRNAs as big regulators of neural stem/progenitor cell proliferation, differentiation and migration: a potential treatment for stroke. Curr Pharm Des. 2017;23 (15):2252-2257. doi:10.2174/1381612823666170228124657

14. Gao X, Zhang W, Yuan J, Xu X, He J, Fu C. [Association of microRNA101 expression with clinicopathologic features and prognosis in colorectal cancer]. Zhonghua Wei Chang Wai Ke Za Zhi. 2015;18(4):365-369. Chinese.

15. Zhu L, Chen Y, Nie K, Xiao Y, Yu H. MiR-101 inhibits cell proliferation and invasion of pancreatic cancer through targeting STMN1. Cancer Biomark. 2018;23(2):301-309. doi:10.3233/CBM-181675

16. Yi J, Huang WZ, Wen YQ, Yi YC. Effect of miR-101 on proliferation and oxidative stress-induced apoptosis of breast cancer cells via $\mathrm{Nrf} 2$ signaling pathway. Eur Rev Med Pharmacol Sci. 2019;23 (20):8931-8939.

17. Ouyang J, Pan X, Lin H, Hu Z, Xiao P, Hu H. GKN2 increases apoptosis, reduces the proliferation and invasion ability of gastric cancer cells through down-regulating the JAK/STAT signaling pathway. Am J Transl Res. 2017;9(2):803-811.
18. Wu X, Tao P, Zhou Q, et al. IL-6 secreted by cancer-associated fibroblasts promotes epithelial-mesenchymal transition and metastasis of gastric cancer via JAK2/STAT3 signaling pathway. Oncotarget. 2017;8(13):20741-20750. doi:10.18632/oncotarget.15119

19. Zhao JM, Cheng W, He XG, Liu YL, Wang FF, Gao YF. Long non-coding RNA PICART1 suppresses proliferation and promotes apoptosis in lung cancer cells by inhibiting JAK2/STAT3 signaling. Neoplasma. 2018;65(5):779-789. doi:10.4149/neo_2018_171130N778

20. Tian YS, Zhong D, Liu QQ, et al. Upregulation of miR-216a exerts neuroprotective effects against ischemic injury through negatively regulating JAK2/STAT3-involved apoptosis and inflammatory pathways. J Neurosurg. 2018;130(3):977-988. doi:10.3171/2017.5.JNS163165

21. Zhang Y, Liu J, Yang B, et al. Ginkgo biloba extract inhibits astrocytic lipocalin-2 expression and alleviates neuroinflammatory injury via the JAK2/STAT3 pathway after ischemic brain stroke. Front Pharmacol. 2018;9:518. doi:10.3389/fphar.2018.00518

22. Ziu M, Fletcher L, Rana S, Jimenez DF, Digicaylioglu M. Temporal differences in microRNA expression patterns in astrocytes and neurons after ischemic injury. PLoS One. 2011;6(2):e14724. doi:10.1371/ journal.pone. 0014724

23. Yin KJ, Deng Z, Huang H, et al. miR-497 regulates neuronal death in mouse brain after transient focal cerebral ischemia. Neurobiol Dis. 2010;38(1):17-26. doi:10.1016/j.nbd.2009.12.021

24. Shi GD, OuYang YP, Shi JG, Liu Y, Yuan W, Jia LS. PTEN deletion prevents ischemic brain injury by activating the mTOR signaling pathway. Biochem Biophys Res Commun. 2011;404(4):941-945. doi:10.1016/j.bbrc.2010.12.085

25. Zhou L, Li F, Xu HB, et al. Treatment of cerebral ischemia by disrupting ischemia-induced interaction of nNOS with PSD-95. Nat Med. 2010;16(12):1439-1443. doi:10.1038/nm.2245

26. Longa EZ, Weinstein PR, Carlson S, Cummins R. Reversible middle cerebral artery occlusion without craniectomy in rats. Stroke. 1989;20 (1):84-91. doi:10.1161/01.STR.20.1.84

27. Johnston SC, Mendis S, Mathers CD. Global variation in stroke burden and mortality: estimates from monitoring, surveillance, and modelling. Lancet Neurol. 2009;8(4):345-354. doi:10.1016/S14744422(09)70023-7

28. Mello EA, Cohen LG, Monteiro Dos Anjos S, et al. Increase in short-interval intracortical facilitation of the motor cortex after low-frequency repetitive magnetic stimulation of the unaffected hemisphere in the subacute phase after stroke. Neural Plast. 2015;2015:407320. doi:10.1155/2015/407320

29. Zhao J, Mou Y, Bernstock JD, et al. Synthetic oligodeoxynucleotides containing multiple telemeric TTAGGG motifs suppress inflammasome activity in macrophages subjected to oxygen and glucose deprivation and reduce ischemic brain injury in stroke-prone spontaneously hypertensive rats. PLoS One. 2015;10(10):e140772. doi:10.1371/journal.pone.0140772

30. Chai Z, Yin X, Chen J, et al. MicroRNA-101 modulates cisplatin chemoresistance in liver cancer cells via the DNA-PKcs signaling pathway. Oncol Lett. 2019;18(4):3655-3663.

31. Li XN, Wang ZJ, Ye CX, Zhao BC, Huang XX, Yang L. Circular RNA circVAPA is up-regulated and exerts oncogenic properties by sponging miR-101 in colorectal cancer. Biomed Pharmacother. 2019;112:108611. doi:10.1016/j.biopha.2019.108611

32. Chen X. MiR-101 acts as a novel bio-marker in the diagnosis of bladder carcinoma. Medicine. 2019;98(26):e16051. doi:10.1097/ MD.0000000000016051

33. Lin YL, Zou ZK, Su HY, Huang YQ. [Expression of MiR101 and EZH2 in Patients with Mantle Cell Lymphoma and Its Clinical Significance]. Zhongguo Shi Yan Xue Ye Xue Za Zhi. 2019;27 (3):820-826. Chinese.

34. Dong X, Liu Y. Expression and significance of miR-24 and miR-101 in patients with advanced gastric cancer. Oncol Lett. 2018;16 (5):5769-5774. 
35. Jiang W, Pan JJ. Down-regulated serum microRNA-101 is associated with aggressive progression and poor prognosis of cervical cancer. J Gynecol Oncol. 2017;28(6):e75.

36. Wang Y, Zhang S. Berberine suppresses growth and metastasis of endometrial cancer cells via miR-101/COX-2. Biomed Pharmacother. 2018;103:1287-1293. doi:10.1016/j.biopha.2018.04.161

37. Barbato C, Pezzola S, Caggiano C, et al. A lentiviral sponge for miR-101 regulates RanBP9 expression and amyloid precursor protein metabolism in hippocampal neurons. Front Cell Neurosci. 2014;8:37. doi:10.3389/fncel.2014.00037

38. Satriotomo I, Bowen KK, Vemuganti R. JAK2 and STAT3 activation contributes to neuronal damage following transient focal cerebral ischemia. $J$ Neurochem. 2006;98(5):1353-1368. doi:10.1111/j.14714159.2006.04051.x

39. Zhou K, Chen J, Wu J, et al. Atractylenolide III ameliorates cerebral ischemic injury and neuroinflammation associated with inhibiting JAK2/STAT3/Drp1-dependent mitochondrial fission in microglia. Phytomedicine. 2019;59:152922. doi:10.1016/j.phymed.2019.152922

40. Gao GS, Li Y, Zhai H, et al. Humanin analogue, S14G-humanin, has neuroprotective effects against oxygen glucose deprivation/reoxygenation by reactivating Jak2/Stat3 signaling through the PI3K/ AKT pathway. Exp Ther Med. 2017;14(4):3926-3934. doi:10.3892/ etm.2017.4934
41. Wang X, Kan J, Han J, Zhang W, Bai L, Wu H. LncRNA SNHG16 functions as an oncogene by sponging MiR-135a and promotes JAK2/STAT3 signal pathway in gastric cancer. $J$ Cancer. 2019;10 (4):1013-1022. doi:10.7150/jca.29527

42. Zhou W, Bi X, Gao G, Sun L. miRNA-133b and miRNA-135a induce apoptosis via the JAK2/STAT3 signaling pathway in human renal carcinoma cells. Biomed Pharmacother. 2016;84:722-729. doi:10.1016/j.biopha.2016.09.074

43. Wang L, Zhang H. microRNA-146a promotes growth of acute leukemia cells by downregulating ciliary neurotrophic factor receptor and activating JAK2/STAT3 signaling. Yonsei Med J. 2019;60 (10):924-934.

44. Wang L, Zhuang L, Rong H, et al. MicroRNA-101 inhibits proliferation of pulmonary micro vascular endothelial cells in a rat model of hepatopulmonary syndrome by targeting the JAK2/STAT3 signaling pathway. Mol Med Rep. 2015;12:8261-8267. doi:10.3892/ mmr.2015.4471

\section{Publish your work in this journal}

Neuropsychiatric Disease and Treatment is an international, peerreviewed journal of clinical therapeutics and pharmacology focusing on concise rapid reporting of clinical or pre-clinical studies on a range of neuropsychiatric and neurological disorders. This journal is indexed on PubMed Central, the 'PsycINFO' database and CAS, and is the official journal of The International Neuropsychiatric Association (INA). The manuscript management system is completely online and includes a very quick and fair peer-review system, which is all easy to use. Visit http://www.dovepress.com/testimonials.php to read real quotes from published authors. 